\title{
Acupuntura e promoção de saúde: possibilidades no serviço público de saúde*
}

Maria Elisa Rizzi Cintra ${ }^{1}$ Regina Figueiredo²

CINTRA, M.E.R.; FIGUEIREDO, R. Acupuncture and health promotion: possibilities in public health services. Interface - Comunic., Saude, Educ., v.14, n.32, p.139-54, jan./mar. 2010.

The objective of the present study was to analyze the possible contributions of acupuncture applied within public health services in the municipality of São Paulo, for health promotion. A qualitative research approach was used, with in-depth interviews. The results showed that acupuncture practiced within the public health services enabled interdisciplinarity, thereby integrating individuals' self-perception and their perception of context and allowing changes in the primary causes of diseases. Thus, contact with acupuncture as practiced in the health services of the municipal network of São Paulo may be able to contribute towards developing health promotion actions.

Keywords: Acupuncture. Health promotion. Single Health System.
O presente estudo objetivou analisar as possíveis contribuições da Acupuntura aplicada nos serviços públicos de saúde do município de São Paulo para a Promoção da Saúde. Foi utilizada a abordagem qualitativa de pesquisa, com uso de entrevistas em profundidade. Os resultados apontam que a Acupuntura praticada nos serviços públicos permite um trânsito interdisciplinar integrando a percepção do indivíduo sobre si mesmo e seu contexto, possibilitando mudanças nas causas primárias das doenças. Assim, o contato com a Acupuntura praticada nos serviços de saúde da rede municipal de São Paulo mostra-se passível de contribuir para o desenvolvimento de ações de Promoção de Saúde.

Palavras-chave: Acupuntura. Promoção de saúde. Sistema Único de Saúde.
*Texto inédito, com aprovação do Comitê de Ética/ Sisnep CAAE0046.0.162.253-07.

${ }^{1}$ Mestranda, Programa de Pós-Graduação em Saúde Coletiva, Departamento de Medicina Preventiva, Universidade Federal de São Paulo. Rua dos Pinheiros, 1474, apto. 604. São Paulo, SP, Brasil. 05.422-002.

elisa.rizzi@hotmail.com 2 Instituto de Saúde Secretaria de Estado da Saúde de São Paulo. 


\section{Introdução}

Em especial após a década de 1960, o movimento internacional de contracultura vem agregando ao imaginário brasileiro tradições provenientes de culturas orientais. Tal movimento sugeria um novo estilo de vida às pessoas, incorporando tendências naturalistas e de afinidade com as civilizações do Oriente. No âmbito econômico, em sentido contrário à industrialização e consumos desenfreados, o movimento preconizava os conceitos de desenvolvimento sustentável e de qualidade de vida como uma forma mais equilibrada de interação com o meio social e ambiental, pressupondo a valorização do corpo, da saúde, da natureza, do prazer e, especialmente, das emoções positivas (Queiroz, 2006).

A maior parte destas tradições adota uma postura "vitalista" diante do corpo, da saúde e da doença, que tem, como aspecto teórico fundamental, a ideia de que a "energia" organiza a matéria, e não vice-versa, com ênfase no estado geral do doente e não mais na doença; numa perspectiva integradora e nãoorganicista, que interpreta a doença como um desequilíbrio interno, e não como resultado de invasões de agentes patogênicos (Queiroz, 2000). A doença, assim, representa as manifestações sintomáticas de desequilíbrio, que são vistas como sintomas necessários, provenientes de causas mais profundas, que abrangem o indivíduo e seu modo de vida em sua totalidade. Assim, as propostas de saúde influenciadas pelo contato com o Oriente se caracterizam por serem, não intervencionistas, mas focadas no indivíduo, seu meio ambiente e sua experiência de vida (Queiroz, 2006). Dentre elas está a Medicina Tradicional Chinesa-MTC ${ }^{3}$.

A Acupuntura é uma técnica de intervenção terapêutica da MTC que adota essa postura vitalista, uma vez que se fundamenta no primado da energia sobre a matéria, do doente sobre a doença, e na ideia de "tipos constitucionais humanos", características de pessoas com determinados padrões físicos, estruturais, psicológicos e de comportamento (Hicks, Hicks, Mole, 2007).

No Brasil, a prática da Acupuntura foi introduzida na tabela do Sistema de Informação Ambulatorial - SIA/SUS em 1999, através da Portaria no 1230/GM (Brasil, 1999), e sua prática reforçada pela Portaria 971, publicada pelo Ministério da Saúde em 2006, que aprovou a Política Nacional de Práticas Integrativas e Complementares no Sistema Único de Saúde. Este último documento define que a mesma pode ser aplicada junto aos sistemas médicos complexos. Esse documento define que, no SUS - Sistema único de Saúde, sejam integrados abordagens e recursos que busquem estimular os mecanismos naturais de prevenção de agravos e de recuperação da saúde, sobretudo, os com ênfase na escuta acolhedora, no desenvolvimento do vínculo terapêutico e na integração do ser humano com o meio ambiente e com a sociedade (Brasil, 2006).

Tal determinação legal permitiu que alguns serviços de saúde pública brasileiros integrassem a prática da Acupuntura (Nascimento, 2006). No município de São Paulo, isso vem ocorrendo, mais amplamente, desde 2000, por iniciativa de profissionais do Hospital do Servidor Público Municipal, do Hospital e Maternidade Escola da Vila Nova Cachoeirinha, da Unidade Básica de Saúde da Vila Progresso, na Brasilândia, dos Centros de Referência da Saúde do Trabalhador de Santo Amaro e da Freguesia do Ó e do Centro de Saúde Escola da Faculdade de Saúde Pública.

Em 2001, a Secretaria Municipal da Saúde de São Paulo, com o objetivo de difundir suas distintas modalidades em todos os serviços públicos de saúde do município, criou a Área Técnica das Medicinas Tradicionais e Práticas Integrativas em Saúde. Até o final de 2004, mais de mil funcionários passaram por processos de capacitação, resultando na integração de, pelo menos, uma modalidade de

\author{
${ }^{3}$ A partir desta \\ ocorrência, o termo \\ Medicina Tradicional \\ Chinesa aparecerá no \\ texto com a sigla MTC.
}


${ }^{4}$ Mais popularmente conhecido no Brasil, na Grafia Wade-Giles, por "Tai Chi Chuan".

Este artigo adotará a padronização dos termos chineses na grafia Pin Yin.

${ }^{5}$ As referências de tempo nos estudos sobre a China são usualmente feitas através das dinastias (Souza, 2008). prática integrativa oriunda da MTC (entre elas TÀl Jí QUÀN ${ }^{4}$, LIANG GONG, meditação, TUÍ-NA e Acupuntura), em cerca de trezentas unidades de saúde do município de São Paulo. Desse total de serviços, sessenta integraram especificamente o recurso da Acupuntura (Brasil, 2002), número que, no entanto, sofreu uma regressão até os dias atuais.

O estudo aqui apresentado teve como objetivo analisar as possíveis contribuições da Acupuntura aplicada nos serviços públicos de saúde do município de São Paulo para a Promoção da Saúde. Para sua execução foi utilizada a abordagem qualitativa de pesquisa, com uso de entrevistas em profundidade.

\section{Medicina tradicional chinesa, Acupuntura e promoção de saúde}

Dentre os autores do Ocidente Contemporâneo que estudaram a fundo as tradições, a sociedade, a filosofia e história chinesa, destacam-se: Marcel Granet e Paul Unschuld. Granet foi um dos primeiros etnólogos a estudar a China pelos métodos sociológicos, a partir de textos clássicou chineses.

Deixando um legado de tratados minuciosos sobre a civilização e o pensamento chineses:
As idéias conjuntas de Ordem, Totalidade e Eficácia dominam o pensamento dos chineses. Eles não se preocupam em distinguir reinos da natureza. Toda realidade é total em si. Todo universo é como o Universo. A matéria e o espírito não aparecem como dois mundos opostos. Não confere ao homem um lugar à parte [...] a não ser na medida em que, possuindo uma posição na sociedade, é digno de colaborar na manutenção da ordem social, fundamento e modelo da ordem universal. [...]. Essas idéias coadunam-se com uma representação do mundo que se caracteriza não pelo antropocentrismo, mas pela predominância da noção de autoridade social. (Granet, 1997, p.211)

Unschuld (1985) aponta a visão não-linear e plural dos sistemas de saúde chineses. Preocupado com as distorções frequentemente encontradas nas traduções da literatura em MTC, ele sugere uma abordagem histórica e sistemática baseada em fontes antigas disponíveis na China.

Ao apresentar o modelo de evolução da Medicina Chinesa em uma longa escala de tempo desde a dinastia ${ }^{5}$ SHANG (1751-112 a.C.) até o período atual conhecido como República Popular da China, iniciado em 1949, o autor demonstra como foram ocorrendo as mudanças de paradigmas na MTC, que inicialmente pautada num modelo oracular e sem diferenciação clara entre as funções do sacerdote e do terapeuta, hoje se aproxima cada vez mais da Medicina Ocidental ou da Racionalidade Biomédica (Souza, 2008).

Segundo Luz e Souza (2009), a Medicina Clássica Chinesa - MCC foi sistematizada durante a dinastia HÀN (206 a.C.-220 d.C.). Houve, nesse período, uma síntese das concepções da cosmologia e da sociologia dos chamados "filósofos" chineses com concepções específicas do saber médico, que formaram as dimensões da doutrina médica, morfologia, dinâmica vital, diagnose e terapêutica. Destaca-se, nesse modelo, o HUÁNG Dì NÈ JING, o Livro do Imperador Amarelo, e NAN JING, O Clássico das Dificuldades, nos quais foram sistematizados, num modelo coerente, a teoria dos meridianos (JING MÀI) e dos órgãos e vísceras (ZÀNG FU) e a teoria dos fatores patogênicos, a qual passa a ser a principal forma de explicar o adoecimento na dimensão da doutrina médica - e a prática da acupuntura aparece integrada a este modelo (Luz, Souza, 2009). 
Ainda de acordo com Luz e Souza (2009), o declínio da Medicina Clássica se iniciou na dinastia QING (1644-1912), já sob influência da cultura ocidental em expansão, os valores da cultura chinesa foram lentamente sendo transformados pelos valores ocidentais. A reconstrução da Medicina Chinesa teve início após a revolução comunista de 1949, no processo de construção da República Popular de China, uma vez que seus dirigentes tinham como objetivo resgatar parte da cultura tradicional criando uma síntese com a ciência e os valores modernos; adequando-se aos valores e ideologia da China comunista, surge então a MTC.

Nos últimos quarenta anos, a escola de pensamento denominada MTC alcançou uma posição hegemônica na República Popular da China, bem como em diversos países do ocidente. Sendo uma das práticas terapêuticas mais divulgadas da Medicina Chinesa, a Acupuntura se apresenta como uma opção terapêutica disponível em qualquer metrópole do mundo ocidental (Souza, 2008).

A MTC tem por base a integração e interação entre o ser humano e a natureza, visando o equilíbrio geral das pessoas (Alvarenga et al., 2004). O organismo é visto como um sistema energético e funcional e as doenças vistas como desequilíbrios energéticos, ou "quebra" na harmonia das funções orgânicas. Os fenômenos que ocorrem nos órgãos são explicados por meio de síndromes (conjunto de fatores patológicos de origem interna ou externa ao organismo) que revelam como a base energética da existência e a expressão da matéria, a força vital (Qì), está circulando no sistema de órgãos e vísceras da pessoa (ZÀNG FU).

Os indicadores das síndromes são verificados por oito critérios de diagnóstico: quanto à localização: (1) interno (profundo) ou (2) externo (superficial); quanto à natureza: (3) frio ou (4) calor; quanto à intensidade: (5) vazio (deficiência) ou (6) plenitude (excesso); quanto ao princípio: (7) yin ou (8) yang. Também importa, no diagnóstico, a análise do meio em que a pessoa se encontra e as condições de vida, sendo fundamentais, o aspecto emocional, os hábitos alimentares, os hábitos sexuais e a atividade física (Luz, 1993).

O "fluxo de energia" no corpo é analisado com base nos cinco elementos existentes na natureza: fogo, terra, metal, água e madeira. Esses cinco elementos são vistos como responsáveis por uma série de relações e ciclos entre si, que explicariam os fenômenos vitais do corpo humano (Luz, 2006). Assim, o estado de saúde, nesta concepção, corresponde a um estado de equilíbrio do corpo, da mente e da espiritualidade e a manifestação da plena interação destes.

No caso da Acupuntura, o método consiste na estimulação (com agulhas, eletricidade, esferas ou sementes) de pontos específicos da pele onde se localizam os padronizados canais definidos como "meridianos", onde passaria a força vital (Ql̀). Esses meridianos estão, cada um, em vinculação direta com um respectivo sistema fisiológico e/ou mental da pessoa. Por isso, estimulando (tonificando) ou pressionando (sedando/ dispersando) tais pontos, altera-se a circulação de energia vital (Qì) e o fluxo de sangue (XUĖ) do organismo (Fundamentos, 1995).

A MTC configura-se como uma racionalidade médica diferente da Biomedicina. A categoria criada por Madel Luz (1992) postula que um sistema terapêutico complexo é uma "racionalidade médica" quando engloba: 1 uma cosmologia; 2 uma morfologia; 3 uma dinâmica vital (ou Fisiologia); 4 uma doutrina médica (causas, efeitos e definições do adoecer são explicados); 5 um sistema de diagnose e 6 uma terapêutica.

Como a Acupuntura faz parte dessa racionalidade integradora e vitalista da Medicina Tradicional Chinesa, acredita-se que seu tratamento tenha a possibilidade de promover o contato e o aprendizado de novas formas de se pensar o corpo, a saúde e a doença, viabilizando ações promotoras de saúde.

A expressão Promoção da Saúde surgiu em 1945 (Pereira et al., 2000) para descrever uma das quatro tarefas básicas da Medicina relativas à prevenção de doenças, ao tratamento de doentes e à reabilitação. As ações de Promoção da Saúde englobam a promoção de condições de vida decentes, boas condições de trabalho, educação, exercício físico e descanso.

Em 1966, Leavell e Clark, ao sistematizarem o modelo de história natural de doença, incluíram a Promoção de Saúde no primeiro dos três níveis de ações de prevenção à saúde: 1 ações de promoção, prevenção e de profilaxia; 2 ações de diagnóstico e tratamento; e 3 ações de reabilitação (Leavell, Clark, 1976). Nesse modelo, as atividades a serem realizadas para promover a saúde incluiriam desde a boa nutrição, ao atendimento às necessidades afetivas e atividades de educação para a saúde. 
Mais tarde, em 1974, Marc Lalonde procurou ultrapassar as limitações nas definições das ações de Promoção da Saúde centradas na assistência médica, privilegiando as ações voltadas a "fatores particulares" (como a modificação de hábitos, do estilo de vida e dos comportamentos individuais não-saudáveis). Sua contribuição repercutiu durante toda a década de 1970, orientando práticas de Promoção da Saúde em diversos países da América do Sul e Europa (Sícoli, Nascimento, 2003).

Na década de 1980, o termo Promoção da Saúde passou a se destacar no campo da Saúde Pública, ao ser introduzido oficialmente pela Organização Mundial de Saúde em 1984, passando a explicitar tanto a determinação social, política e econômica sobre a saúde, como a necessidade de atuação junto a esses determinantes (Sícoli, Nascimento, 2003). Essa visão foi reforçada pela Declaração de AlmaAta (World Health Organization - WHO, 1978) e pela Carta de Otawa (WHO, 1986), esta última elaborada na $1^{\text {a }}$ Conferência Internacional sobre a Promoção de Saúde. A Carta de Otawa define que a Promoção da Saúde é um processo de capacitação da comunidade para atuar na melhoria de sua qualidade de vida e saúde, visando atingir um estado de completo bem-estar físico, mental e social (WHO, 1986).

Posteriormente, em 1998, diante do cenário de crescentes preocupações com os princípios norteadores das ações de Promoção, a Organização Mundial de Saúde (OMS) definiu sete princípios norteadores para as políticas e as ações de Promoção de Saúde: 1 a concepção holística, 2 a intersetorialidade, 30 empoderamento, 4 a participação social, 5 a equidade, 6 as ações multiestratégicas, e 7 a sustentabilidade (WHO, 1998).

Os mesmos princípios são contemplados pela Política Nacional de Promoção da Saúde (PNPS) brasileira, criada em 2005:

Promover a qualidade de vida e reduzir vulnerabilidade e riscos à saúde relacionados aos seus determinantes e condicionantes - modos de viver, condições de trabalho, habitação, ambiente, educação, lazer, cultura, acesso a bens e serviços essenciais. (Brasil, 2005, p.14)

\section{Promoção de saúde: um conceito positivo}

Segundo Lefèvre e Lefèvre (2004), admitir a possibilidade de promover saúde implica, necessariamente, o rompimento da visão da doença como fatalidade e assumir que a doença não é uma fatalidade e que a saúde não é mera resposta a ser reproduzida. Os autores consideram o modo negativo de conceber saúde como inadequado, pois este considera a saúde apenas como a ausência de doenças, revelando uma visão fragmentada do ser humano, baseada unicamente no modelo biomédico.

De acordo com Camargo Jr. (2003), a biomedicina tem suas origens no surgimento da ciência moderna. A partir do Renascimento, progressivamente, foram sendo instauradas as bases da racionalidade mecanicista clássica e do positivismo, que têm como expoentes René Descartes e Auguste Comte, respectivamente. Nesse mesmo momento histórico nasce a clínica (Foucault, 2008), que, embasada nesta episteme, impregna o imaginário médico (mesmo séculos mais tarde) na racionalidade mecânica clássica (Camargo Jr., 2003), paralelamente ao desenvolvimento das Ciências Naturais.

A ótica mecanicista pode ser delineada em três proposições básicas: 1 Seu caráter generalizante, ou seja, dirigir-se à produção de discursos de validade universal; 2 Seu caráter mecanicista, ou seja, a naturalização das máquinas produzidas pelo Homem, ao mesmo tempo em que passa a ver o universo como uma gigantesca máquina subordinada a princípios de causalidade lineares traduzíveis em mecanismos; 3 Seu caráter analítico, que pressupõe o isolamento das partes e o funcionamento do todo dado, necessariamente, pela soma das partes, portanto, buscam-se "leis gerais" (Camargo Jr., 2005).

A Biomedicina se origina a partir da anatomoclínica; é uma medicina do corpo, das lesões e das doenças. A perspectiva de encontrar-se a essência da doença a partir do exame empírico de lesões delineia todo um novo campo epistemológico; com isso, apresenta-se nova concepção de doença, as quais não mais são vistas como um fenômeno vital, mas como a expressão de lesões celulares; assim, a doença passa a ser a categoria central do saber e da prática médica. Para Madel Luz, a Medicina 
Ocidental torna-se definitivamente uma ciência das doenças, contrapondo-se à concepção médica da "arte de curar" (Camargo Jr., 2003, p.32).

A doutrina biomédica traz implícita a ideia de que as doenças são objetos com existência autônoma; a perspectiva de uma "normalidade" é caracterizada por sua oposição à "patologia", e a própria noção de saúde é definida como ausência de doença, por isso é tida como uma concepção negativa (Canguilhem apud Camargo, 2005, p.181).

Em resposta a esta concepção "negativa", Lefèvre e Lefèvre (2004) defendem que a concepção dada pela área da Promoção da Saúde, ao contrário, é "positiva", pois é ampliada, integrada, complexa e intersetorial. Ao relacionar a saúde aos recursos sociais e pessoais, assim como às capacidades físicas, objetivando o bem-estar global, a área da Promoção da Saúde termina por ir além do setor Saúde.

Assim, os autores propõem à Promoção de Saúde um papel de interferência, reunindo o conhecimento historicamente acumulado do processo saúde/doença como indicador das necessidades de intervenção com o objetivo de atingir as causas básicas da doença e, não apenas, evitar que elas se manifestem nos indivíduos e nas coletividades.

Dentre as recomendações de ações de Promoção à Saúde, a Organização Mundial de Saúde (OMS) estimula a integração das medicinas tradicionais nos sistemas de saúde, procurando promover estratégias de atuação mais amplas, que integrem, ao mesmo tempo, os requisitos de segurança, eficácia, qualidade, uso racional e acesso (WHO, 1998). Entre essas técnicas, a OMS cita a fitoterapia, a homeopatia, e, também, a Medicina Tradicional Chinesa, que inclui a terapêutica da Acupuntura.

\section{Metodologia}

O presente estudo foi realizado utilizando-se a abordagem qualitativa de pesquisa. Em 2007, os serviços de saúde da região centro-oeste do município de São Paulo totalizavam 44 unidades básicas de saúde. Dentre eles, foram selecionados os quatro serviços municipais e com carga horária de atendimento mínimo de 4 horas semanais em Acupuntura.

Os profissionais entrevistados foram escolhidos por serem os responsáveis pelo atendimento em Acupuntura nas unidades, por meio do contato direto com os mesmos, após autorização das respectivas coordenações dos serviços. Os usuários entrevistados foram convidados entre aqueles que estavam fazendo ou que já haviam feito tratamento com Acupuntura nesses mesmos serviços, em número que obedeceu ao critério de saturação de informações.

$\mathrm{Na}$ abordagem dos participantes foram adotados todos os procedimentos de ética em pesquisa, orientados pela resolução 196/96 (CNS, 1996), além da submissão e aprovação da pesquisa pelo Comitê de Ética em Pesquisa.

\section{Resultados obtidos e discussão}

Foram realizadas 13 entrevistas em profundidade, sendo quatro com profissionais e nove com usuários de Acupuntura. As entrevistas tiveram seus conteúdos analisados individualmente, e, depois, em seu conjunto, organizados por categorias temáticas por meio da análise transversal de conteúdo ${ }^{6}$.

\footnotetext{
${ }^{6} \mathrm{~A}$ análise transversal consiste em descobrir progressivamente as analogias e/ou complementos entre as diferentes experiências e reflexões presentes num conjunto de arquivos, para extrair daí os elementos comuns.
} 
Os profissionais entrevistados nesta pesquisa possuíam formação anterior na área da biomedicina, pelo menos seis horas de trabalho semanal como acupunturista num serviço público, e a maioria com mais de dez anos de formação na área, como demonstra o quadro a seguir:

Quadro 1. Perfil dos profissionais de Acupuntura entrevistados entre os meses de junho e setembro de 2007.

\begin{tabular}{|c|c|c|c|c|c|c|}
\hline \multirow[t]{2}{*}{ Entrevistado } & \multirow[t]{2}{*}{ Sexo } & \multirow{2}{*}{$\begin{array}{c}\text { Formação } \\
\text { inicial }\end{array}$} & \multirow{2}{*}{$\begin{array}{l}\text { Tempo de formado } \\
\text { em Acupuntura } \\
\text { (anos) }\end{array}$} & \multirow{2}{*}{$\begin{array}{l}\text { Instituição de } \\
\text { formação }\end{array}$} & $\begin{array}{l}\text { Carga horária } \\
\text { total no serviço }\end{array}$ & $\begin{array}{c}\text { Carga } \\
\text { horária como } \\
\text { Acupunturista }\end{array}$ \\
\hline & & & & & \multicolumn{2}{|c|}{ (horas por semana) } \\
\hline P1 & $M$ & Sanitarista & 13 & Pública & 30 & 30 \\
\hline P2 & M & Clínico & 30 & Pública/ Particular & 30 & 30 \\
\hline P3 & $\mathrm{F}$ & Pediatra & 3 & Pública/ Particular & 40 & 6 \\
\hline P4 & $\mathrm{F}$ & Sanitarista & 20 & Pública/ Particular & 40 & 6 \\
\hline
\end{tabular}

Fonte: Entrevista com os profissionais de Acupuntura.

Os usuários entrevistados são todos adultos, e têm escolaridade até o $2^{\circ} \mathrm{Grau}$. Além disso, são em grande maioria do sexo feminino, oito entre nove, e buscaram espontaneamente o tratamento com Acupuntura, como demonstra o quadro:

Quadro 2. Perfil dos usuários de Acupuntura entrevistados entre os meses de junho e setembro de 2007.

\begin{tabular}{|c|c|c|c|c|c|c|}
\hline \multirow{2}{*}{ Unidade } & Entrevistado & Sexo & Idade & $\begin{array}{c}\text { Escolaridade } \\
\text { (estágio/completo ou } \\
\text { incompleto) }\end{array}$ & $\begin{array}{c}\text { Recebe } \\
\text { tratamento com } \\
\text { Acupuntura }\end{array}$ & $\begin{array}{c}\text { Como entrou em } \\
\text { contato com a } \\
\text { Acupuntura }\end{array}$ \\
\hline \multirow{2}{*}{$\mathrm{A}$} & $\mathrm{U} 1$ & $\mathrm{~F}$ & 77 & $2^{\circ} \mathrm{G}-\mathrm{C}$ & Atualmente & Espontaneamente \\
\cline { 2 - 7 } & $\mathrm{U} 2$ & $\mathrm{~F}$ & 50 & $1^{\circ} \mathrm{G}-\mathrm{I}$ & Atualmente & Encaminhamento \\
\hline \multirow{3}{*}{$\mathrm{B}$} & $\mathrm{U} 3$ & $\mathrm{~F}$ & 42 & $2^{\circ} \mathrm{G}-\mathrm{C}$ & Anteriormente & Encaminhamento \\
\cline { 2 - 7 } & $\mathrm{U} 4$ & $\mathrm{~F}$ & 35 & $2^{\circ} \mathrm{G}-\mathrm{C}$ & Anteriormente & Espontaneamente \\
\hline \multirow{2}{*}{$\mathrm{C}$} & $\mathrm{U} 5$ & $\mathrm{M}$ & 35 & $2^{\circ} \mathrm{G}-\mathrm{I}$ & Atualmente & Espontaneamente \\
\cline { 2 - 7 } & $\mathrm{U} 6$ & $\mathrm{~F}$ & 60 & $2^{\circ} \mathrm{G}-\mathrm{I}$ & Atualmente & Encaminhamento \\
\hline \multirow{2}{*}{$\mathrm{D}$} & $\mathrm{U} 8$ & $\mathrm{~F}$ & 55 & $2^{\circ} \mathrm{G}-\mathrm{I}$ & Atualmente & Encaminhamento \\
\cline { 2 - 7 } & $\mathrm{U} 8$ & $\mathrm{~F}$ & 62 & $2^{\circ} \mathrm{G}-\mathrm{C}$ & Atualmente & Espontaneamente \\
\hline
\end{tabular}

Fonte: Entrevista com os profissionais de Acupuntura.

As entrevistas foram divididas com base em três grandes temas de análise:

1 Combinação da Acupuntura com outros tratamentos; 2 Mudanças atribuídas ao tratamento com a Acupuntura; 3 Situação do tratamento com Acupuntura no Serviço Público de Saúde.

1 Combinação da Acupuntura com outros tratamentos de saúde:

Considerou-se como combinação de tratamentos de saúde, sempre que foi relatada a não desistência de um tratamento anterior, ou quando o terapeuta encaminhou o paciente para alguma outra especialidade médica ou prática corporal sem interromper o tratamento com agulhas. 
Foi possível verificar que os acupunturistas trabalham em conjunto com outros tratamentos, da seguinte forma:

Quadro 3. Combinação da Acupuntura com outros tratamentos de saúde segundo os entrevistados, entre os meses de junho e setembro de 2007.*

\begin{tabular}{|c|c|c|c|}
\hline Entrevistados & $\begin{array}{l}\text { Atende ou é atendido } \\
\text { em conjunto com outro } \\
\text { profissional de saúde }\end{array}$ & $\begin{array}{l}\text { Indica ou acata orientação } \\
\text { para alimentação e/ou } \\
\text { prática corporal }\end{array}$ & $\begin{array}{l}\text { Receita ou consome } \\
\text { medicação alopática }\end{array}$ \\
\hline $\mathrm{P} 1$ & Sim & Sim & Não \\
\hline $\mathrm{P} 2$ & Sim & Sim & Sim \\
\hline P3 & Não & Sim & Sim \\
\hline $\mathrm{P} 4$ & Sim & Sim & Sim \\
\hline U1 & Sim & Sim & Sim \\
\hline U2 & Sim & Sim & Sim \\
\hline U3 & Não & Sim & Sim \\
\hline U4 & Não & Não & Não \\
\hline U5 & Sim & Não & Não \\
\hline U6 & $\mathrm{Sim}$ & Sim & Sim \\
\hline U7 & Sim & Sim & Sim \\
\hline U8 & Não & Sim & Sim \\
\hline U9 & Sim & Sim & Sim \\
\hline
\end{tabular}

* As categorias Sim/Não encontradas nas tabelas correspondem, respectivamente, a itens que apareceram na entrevista e a itens que não apareceram. Fonte: Entrevista com os profissionais e usuários de Acupuntura.

Todos os profissionais relataram fazer parceria com profissional de saúde de outra especialidade:

“[...] por encaminhamento interno dos profissionais que atendem aqui. Como esse que você viu, ou por encaminhamento de outro serviço, através de um impresso, um encaminhamento próprio, tal". (P1)

Os usuários entrevistados relataram terem sido orientados pelos terapeutas a exercerem alguma prática corporal. As indicações, em sua maioria, foram para a realização de caminhadas e participação em grupos de ginásticas orientais:

"Faço, faço caminhadas, quando não tá com muita dor, eu faço caminhada e a fisioterapia que é direcionada [...]. Pela fisioterapeuta e pelo acupunturista também". (U3)

“[...] então tem duas vezes por semana um grupo de TÀl Jí [...] então vai fazendo a dobradinha e vai fazendo o acompanhamento do paciente". (P4)

Os resultados indicam também que os profissionais entrevistados incentivam práticas corporais como parte complementar e integrante do tratamento.

Como coloca Luz (2007), na MTC, é com a prática dos exercícios das artes marciais, como o TÀl Jí, que se nota um tipo de mudança pela qual o paciente passa a ser menos dependente de remédios e médicos, tornando-se um agente de cura de si mesmo.

O uso do medicamento alopático sem abandono do tratamento com as agulhas ocorre sempre que necessário, tal como a busca de especialidades médicas como forma de complementação e resolução de enfermidades não contempladas por outra terapêutica. Isso reforça a complementaridade entre as duas racionalidades médicas (biomédica e tradicional chinesa) (Figueiredo, 1999):

“É. Então, mas a gente não descarta a alopatia, tem o hipertenso, o diabético, tem a artrose [...]". (P3) 
Entendendo que, em oposição a uma ciência das doenças biomédica, a MTC valoriza a arte de curar, a terapêutica predomina sobre a diagnose; neste sentido pautam-se, bem como outras medicinas tradicionais, nos seguintes aspectos: a) na reposição do sujeito doente como centro do paradigma médico; b) na re-situação da relação médico-paciente como elemento fundamental da terapêutica; c) na busca de meios terapêuticos, despojados tecnologicamente, menos caros e, entretanto, com igual ou maior eficácia curativa nas situações mais gerais e comuns do adoecimento da população; d) na construção de uma medicina que busque acentuar a autonomia do paciente, e não sua dependência no tocante à relação saúde-enfermidade; e) na afirmação de uma medicina que tenha, como categoria central de seu paradigma, a categoria de Saúde, e não de Doença. Nesse sentido:

Levando-se em consideração o grande e continuado desenvolvimento da tecnologia e da ciência no campo da medicina, e sua incapacidade para reverter tal quadro, a busca de outra racionalidade em saúde por distintos grupos sociais que conformam clientelas de cuidados médicos, e mesmo por profissionais terapeutas, torna-se uma explicação razoável para o sucesso de sistemas terapêuticos regidos por paradigmas distintos dos da medicina científica. (Luz, 2007, p.48)

\section{Mudanças atribuídas ao tratamento com Acupuntura}

Os acupunturistas relataram a ocorrência de mudanças atribuídas ao tratamento com Acupuntura, como:

Quadro 4. Mudanças atribuídas ao tratamento com Acupuntura segundo os entrevistados entre os meses de junho e setembro de 2007.

\begin{tabular}{|c|c|c|c|}
\hline Entrevistados & $\begin{array}{l}\text { Fundamenta-se na } \\
\text { racionalidade médica da } \\
\text { MTC }\end{array}$ & $\begin{array}{l}\text { Houve mudança na } \\
\text { alimentação e/ ou } \\
\text { incentivo ao exercício de } \\
\text { prática corporal }\end{array}$ & $\begin{array}{l}\text { Relata melhora ou } \\
\text { ausência de enfermidades }\end{array}$ \\
\hline P1 & Sim & Sim & Sim \\
\hline $\mathrm{P} 2$ & Sim & Sim & Sim \\
\hline P3 & Sim & Sim & Sim \\
\hline P4 & Sim & Sim & Sim \\
\hline U1 & +-- & Sim & Sim \\
\hline U2 & --- & Sim & Sim \\
\hline U3 & --- & Sim & Sim \\
\hline U4 & --- & Sim & Sim \\
\hline U5 & --- & Não & Sim \\
\hline U6 & --- & Sim & Sim \\
\hline U7 & --- & Sim & Sim \\
\hline U8 & $-\cdots$ & Sim & Sim \\
\hline U9 & $-\cdots$ & Sim & Sim \\
\hline
\end{tabular}

Fonte: Entrevista com os profissionais e usuários de Acupuntura

\subsection{Mudança na racionalidade médica}

Foi possível verificar que a racionalidade que embasa a prática da Acupuntura nos serviços visitados é "holística", ou seja, há uma concepção que engloba a integração do homem com a natureza numa perspectiva de macro e microuniversos, postulando uma integridade biopsicossocial.

“O mais evidente é esta concepção que o corpo não é... Ele não é 'compartimentalizado', ele é uma integração de estruturas, de órgãos, de vísceras, que se complementam [...]. Então este conceito de meridianos, ou de canais de energia que é o princípio de atuação da Acupuntura te, evidentemente, te leva a uma concepção do organismo como um todo. Então isso é básico". (P1) 


\subsection{Mudanças no cotidiano}

Os entrevistados relatam alterações em seu cotidiano. Estas mudanças passaram por diversos aspectos, desde mudanças na disciplina pessoal, no estado emocional e/ou psicológico e comportamental, bem como nas atividades físicas e na alimentação.

Foi verificado que os profissionais de Acupuntura atribuem grande importância às mudanças nos hábitos de vida (alimentação, práticas corporais, respiração, ingestão de água e afetividade) para complementar o tratamento que desenvolvem:

"Ah sim, tanto é que, por exemplo, com leite, a gente sempre fala para tirar o leite, por causa da proteína do leite, que não é bom. E o paciente: mas como ficar sem cálcio, num sei quê? Mas têm as verduras, o cálcio dos vegetais é muito melhor que o do leite, então tenta trocar". (P3)

Assim, o contato com a terapêutica também pode permitir intervenções nas causas primárias dos desequilíbrios que causam as doenças (falta de atividade física, ou da má alimentação), indo de encontro ao que postulam Lefèvre e Lefèvre (2004).

Os usuários dos serviços de Acupuntura entrevistados também relataram a ocorrência de mudanças atribuídas ao tratamento em Acupuntura, em relação à:

\subsection{Melhora ou ausência de enfermidades}

Foi relatada, por todos os usuários, a melhora ou extinção das enfermidades ou quadro clínicos, após o tratamento com Acupuntura, como: perda de peso, ausência de dor (muscular, de cabeça, tendinite, dor nas costas, dor nos ombros, hérnia de disco, bico de papagaio, reumatismo), melhora na deficiência óssea (incluindo osteoporose, artrose), controle da pressão alta, controle da diabetes e melhora no sistema circulatório, entre outras:

“[...] então eu acho que a Acupuntura age em todos os sentidos na depressão, no circulatório, no reumatismo e quando você está assim com o coração magoado, você sabe assim, que até nisso ajuda". (U1)

\section{Situação do tratamento com Acupuntura no serviço público de saúde}

Quando perguntado aos profissionais sobre a inserção da Acupuntura no serviço público de saúde, foi possível identificar as temáticas mais frequentes reveladas:

Quadro 5. Tratamento com Acupuntura no serviço público segundo os entrevistados, entre os meses de junho e setembro de 2007.

\begin{tabular}{|c|c|c|c|c|c|c|c|c|}
\hline \multirow[b]{2}{*}{ Entrevistado } & \multirow{2}{*}{$\begin{array}{l}\text { Verificou } \\
\text { redução de } \\
\text { custos e } \\
\text { consumo de } \\
\text { medicação }\end{array}$} & \multirow[b]{2}{*}{$\begin{array}{c}\text { Citou boa } \\
\text { infraestrutura }\end{array}$} & \multirow{2}{*}{$\begin{array}{c}\text { Considerou } \\
0 \\
\text { tratamento } \\
\text { eficaz }\end{array}$} & \multirow{2}{*}{$\begin{array}{c}\text { Mantém bom } \\
\text { relacionamento } \\
\text { com os outros } \\
\text { profissionais } \\
\text { de saúde }\end{array}$} & \multicolumn{4}{|c|}{ Acesso dos pacientes ao tratamento com Acupuntura } \\
\hline & & & & & $\begin{array}{c}\text { Encaminhamento } \\
\text { profissional }\end{array}$ & $\begin{array}{c}\text { Procura } \\
\text { espontânea }\end{array}$ & $\begin{array}{c}\text { Já } \\
\text { conhecia }\end{array}$ & $\begin{array}{l}\text { Tratamento } \\
\text { anterior } \\
\text { ineficaz }\end{array}$ \\
\hline P1 & Sim & Não & Sim & Sim & Sim & Sim & --. & --- \\
\hline P2 & $\mathrm{Sim}$ & Não & $\mathrm{Sim}$ & $\mathrm{Sim}$ & $\mathrm{Sim}$ & $\mathrm{Sim}$ & -.. & -.- \\
\hline P3 & Sim & Não & $\mathrm{Sim}$ & Não & Sim & Sim & -- & -- \\
\hline $\mathrm{P} 4$ & Sim & Não & Sim & Sim & Sim & Sim & $+\cdots$ & $+\cdots$ \\
\hline U1 & Não & Não & $\mathrm{Sim}$ & 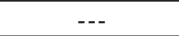 & Não & $\operatorname{Sim}$ & Não & Não \\
\hline U2 & Não & Não & Sim & --- & Sim & Não & Não & Não \\
\hline U3 & Sim & Não & Sim & $-\cdots$ & Sim & Não & Sim & Sim \\
\hline U4 & Sim & Não & Sim & $\cdots$ & Não & Sim & Não & Sim \\
\hline U5 & Não & Não & $\mathrm{Sim}$ & $-\cdots$ & Não & $\mathrm{Sim}$ & Não & Sim \\
\hline U6 & Não & Não & $\mathrm{Sim}$ & -- & Sim & Não & Não & Sim \\
\hline U7 & Não & Não & Sim & - - & Sim & Não & Não & Sim \\
\hline U8 & Sim & Não & Sim & $-\cdots$ & Não & $\operatorname{Sim}$ & Sim & Não \\
\hline U9 & $\mathrm{Sim}$ & Não & $\mathrm{Sim}$ & --- & Não & Sim & Sim & Não \\
\hline
\end{tabular}

Fonte: Entrevista com os profissionais e usuários de Acupuntura. 


\subsection{Motivação para busca de tratamento em Acupuntura}

Foi possível verificar, nos relatos, como os pacientes entraram em contato com o tratamento em Acupuntura nas unidades de saúde e ambulatórios de especialidades. Conseguimos discernir duas principais formas de contato: por encaminhamento e por procura espontânea.

Os relatos dos usuários que foram encaminhados por outros profissionais da área da saúde revelam que variadas especialidades médicas recomendam a Acupuntura como um tratamento complementar às suas práticas:

“Eu comecei, vim do hospital universitário, passava lá porque eu tinha muitas dor, né? Ai fui encaminhada pra aqui, e tô, já fiz, ni mim, fisioterapia, agora tô fazendo Acupuntura". (U2)

Contudo, a maioria dos usuários relatou a procura espontânea pelo tratamento, guiados por diferentes naturezas de motivações, por exemplo, muitos dos usuários buscaram tratamento orientados por outros usuários de Acupuntura conhecidos:

"Aquela que já fez passa pra gente, ai pra mim foi ótimo, então ai recebi aquilo. Ah foi ótimo, ai eu passei pra ela, vamos passando pra frente. Ai isso assim, é como uma bola de neve". (U1)

E outros usuários relataram experiências anteriores de tratamento em Acupuntura com resultados eficazes como sendo motivadoras da busca de um novo tratamento com esta técnica.

“Eu não lembro, foi porque alguém fez, tinham muitas pessoas aqui fazendo, e ai eu fiz. Eu tinha um problema de enxaqueca e depressão e me falaram que era bom, que ajuda, e fui tentar fazer, mas não conhecia". (U5)

Usuários que não resolveram suas queixas com outros tratamentos relataram a busca de outras formas de tratamento, entre elas, a Acupuntura:

“E depois uma artrose e agora osteoporose, tudo relativo ao esqueleto. Então na primeira vez eu tive uma crise muito grande de tendinite nos pulsos, aí comecei a procurar a medicina clássica, cheguei lá e foi indicada cirurgia para os meus pulsos, o pulso direito. Mais aí eu fui pegando opinião, conversando com outras pessoas e aí falaram: por que você não tenta Acupuntura antes da cirurgia?". (U3)

O contato com a Acupuntura também propicia a interação com outras áreas que extrapolam os serviços de saúde, levando as pessoas a parques, a ruas de seu próprio bairro e a integração com seu corpo e com o ambiente espacial e social.

“[...] Além das feiras livres onde você pode comprar alimentos muito mais, em geral mais baratos e pode escolher e os produtos têm mais variedade. Isso é uma coisa que agente estimula muito aqui nessa discussão [...] ai uma da uma sugestão e a outra dá outra. E uma diz: eu compro beterraba e a outra diz que aproveita a folha da beterraba [...]". (P1)

Neste sentido, as falas demonstram ser possível a abrangência apresentada por Alvarenga et al. (2004), que consideram o campo da Saúde Pública amplo e abrangente para contribuições das mais diversas áreas do conhecimento e dos distintos setores da sociedade. Ao mesmo tempo, demonstra que a Promoção de Saúde pode ser interdisciplinar, tal como define a Organização Mundial da Saúde (WHO, 1998), e intersetorial ao possibilitar novos espaços de diálogo.

\subsection{Avaliação do tratamento:}

Foram relatados, pelos profissionais, diversos casos de ausência de dor, melhora de autoestima e 
retrocesso de diversas patologias pelo tratamento com Acupuntura. Assim, verifica-se que a maioria dos resultados destes tratamentos foi eficaz para a melhora ou extinção das enfermidades:

"A Acupuntura me ajudou muito, melhorou bem a minha insônia, e também na depressão [...]". (U6).

"Ajudou, principalmente na obesidade ela foi indicando algumas coisas, o que não comer. Porque eu sou muito ansiosa". (U8)

Os profissionais disseram, também, que a eficácia do tratamento motiva os usuários para adesão ao tratamento com Acupuntura:

"Na verdade, eu sei que é meio difícil, principalmente, tem uns pacientes que tem fibromialgia, e eles vêm cheios de queixas, que é uma doença danada, mas que você vai orientando, batendo na mesma tecla e vendo que melhora mesmo. E então acabam fazendo. Mas muito no perceber que melhora, tá muito ligado a isso, percebeu que melhorou com Acupuntura, alguns conselhos que eu dou, fazendo TÀ̀ Ií melhora, começa a aderir mais". (P2)

\subsection{Relacionamento:}

A respeito da relação com os demais profissionais dos serviços de saúde, os acupunturistas disseram não ter encontrado problemas de convivência. A maioria dos profissionais entrevistados afirma ter um bom relacionamento com colegas de outras especialidades, bem como de outras unidades da região. Foi observado que há troca de informações, experiências e tratamentos realizados em conjunto por diferentes especialidades do setor:

“[...] no geral, agora os médicos também estão indicando, tem hoje a Dra. [...] que tá aqui também, né, do Programa Saúde da Família, [...] então ela também está estimulando". (P4)

Isso, contudo, não foi válido em uma das unidades. Um dos profissionais de Acupuntura relatou ter apresentado grande dificuldade de aceitação e colaboração dos colegas, o que acabou acarretando em seu pedido de demissão:

“[...] E aí foi um problema interno que eu tive lá na UBS. Então, num vou fazer mais, né? [...] Achei que faltou colaboração da recepção, da chefia, porque foi uma coisa assim: não, não dá, mas nem um dia? [...] E de você ouvir umas coisas assim da recepção: Não sei, porque atender Acupuntura, era pra atender 16 que nem os outros médicos, [...] Foi um processo ruim". (P3)

Foi observada também a alteração da hierarquia médico-paciente típica na Medicina Ocidental (Camargo Jr., 2003), pois profissionais relataram aprendizado e reconhecimento de saberes que os pacientes trazem aos atendimentos. A importância da relação terapeuta/paciente foi observada repetida vezes nos serviços de Acupuntura visitados, estabelecendo a confiança e o vínculo mútuos, refletindo a necessidade de mudança nos modelos de organização de assistência à saúde, que hoje possuem caráter curativista e centrados na doença, distanciando o terapeuta do paciente:

"Outro dia, numa das últimas reuniões, uma deu uma receita de aproveitamento de casca de banana, [...] e ai vão surgindo às idéias mais variadas e que sempre alguém aprende alguma coisa com isso. Eu mesmo aprendi com esta história da casca de banana, por exemplo". (P1)

Quanto ao aspecto organizacional, Luz (2007) ressalta a resolutividade que atividades com medicinas alternativas vêm obtendo em programas de serviços públicos de atenção médica ante a demanda da clientela, em relação, sobretudo, às doenças crônicas, tradicionalmente com baixa resolutividade nos sistemas convencionais. Os entrevistados demonstraram que consideram, como positivo, o fato de a terapia da Acupuntura reduzir custos e consumo de medicamentos alopáticos: 
"Eu acho assim, você diminui custos, você diminuí medicamentos, você melhora e beneficia mais os pacientes [...]". (P2)

\subsection{Instrumentalização do paciente para o autocuidado:}

A concepção de "empoderamento" é definida pela Organização Mundial de Saúde (WHO, 1998) como o processo de capacitação dos indivíduos e comunidades para assumirem maior controle sobre os fatores pessoais, socioeconômicos e ambientais que afetam a saúde. Esta preocupação relativa à capacitação dos pacientes para o cuidado com sua própria saúde aparece em diversas passagens das entrevistas realizadas com todos os profissionais:

"[...] tá dentro do protocolo da Acupuntura, do papel da Acupuntura, tornar o paciente o mais possível responsável pela sua saúde e que ele próprio possa também, a partir de alterar práticas hábitos e costumes do cotidiano, evidentemente, melhorar a sua qualidade de vida e, conseqüentemente, da sua saúde". (P1)

Observa-se que o contato com a Acupuntura, nos serviços visitados, incentiva uma postura ativa e uma visão crítica sobre seu próprio corpo. A ampliação da responsabilidade pela própria saúde - o que incluí ações voltadas a "fatores particulares", na busca de hábitos, de estilo de vida mais saudáveis - vem ao encontro da perspectiva apontada em Lalonde (1974) que reforça que não se deve "culpar" a pessoa por sua enfermidade, mas capacitá-la para o autocuidado. As entrevistas revelam a satisfação dos usuários com as orientações para mudança de hábitos, que são seguidas e, em alguns casos, conservadas, como no caso de dois usuários que ainda as seguem após anos do término do tratamento. A prática demonstra potencial para a mudança do papel da pessoa frente a seu tratamento, transformando-a de paciente (passivo) a sujeito (ativo).

De acordo com Luz (2007), em princípio, tais medicinas tendem a propiciar um conhecimento maior do indivíduo a respeito de si mesmo, de seu corpo e de seu psiquismo, com uma consequente busca de maior autonomia ante o seu processo de adoecimento, facilitando um projeto de construção (ou de reconstrução) da própria saúde.

Sem deslocar o modo de conceber, praticar e obter saúde de sua origem no setor saúde, a Promoção de Saúde passa a mobilizar o conhecimento historicamente acumulado no campo sanitário, buscando a saúde em outro lugar, não mais no corpo, nem genericamente em todos os espaços sociais, mas nas relações entre os corpos/mentes doentes e a sociedade. Começa a ser compreendida em seus significados, para daí constituírem objetos de intervenções transformadoras, tal como apontam Lefèvre e Lefévre (2004).

\section{Considerações finais}

A discussão colocada anteriormente apresenta um panorama dos temas mais relevantes levantados pelos participantes desta pesquisa. Esses temas foram divididos em três grandes tópicos de análise: 1 combinação da Acupuntura com outros tratamentos; 2 mudanças atribuídas ao tratamento com a Acupuntura; 3 situação do tratamento com Acupuntura no serviço público de saúde; com a intenção de organizar e facilitar a compreensão do leitor. Todavia, um aspecto importante a ser registrado são os limites da amostra obtida e da própria metodologia qualitativa no que diz respeito às generalizações dos resultados alcançados no caso específico pesquisado. Fizemos a discussão com base em revisões bibliográficas sobre o tema, e especificamente para este grupo de pessoas entrevistadas. Acreditamos que os padrões encontrados podem se repetir em outros serviços e em outras localidades, porém não é possível afirmá-lo. Mas é possível afirmar que profissionais e usuários dos serviços em questão apontam a terapêutica como eficaz para melhoria das enfermidades, e reconhecem que o contato com a terapêutica viabiliza a eliminação ou a diminuição das condições que geram doença.

O conceito de Promoção da Saúde abrange abre um novo leque de possibilidades de ações de 
adesão de cuidados com a saúde (na nutrição, ambiente, saúde metal etc.). Na Promoção da saúde, mais do que na Vigilância à Saúde, é possível intervir não apenas para evitar a doença, mas para estabelecer patamares a serem alcançados na qualidade de vida, reconhecendo o que se quer alcançar. Assim, o arsenal científico e tecnológico disponível na atualidade deve ser direcionado não apenas para corrigir e prevenir doenças, mas para interpretar e tematizar os valores vitais que as patologias observadas estão revelando (Ayres, 2002).

O modelo funcional observado na MTC permite um movimento interpretativo, como propõe Ayres (2002), que revela e critica conteúdos normativos, permitindo um trânsito interdisciplinar e integrando a percepção do indivíduo e de seu contexto. Assim, a Acupuntura praticada nos serviços de saúde da rede municipal de São Paulo mostra-se passível de contribuir para a construção de ações de promoção de saúde, por isso deve ser ampliada para outros serviços e utilizada como exemplo para a incorporação de outras terapias alternativas à prática da biomedicina.

\section{Colaboradores}

Os autores trabalharam juntos em todas as etapas de produção do manuscrito.

\section{Referências}

ALVARENGA, A.T.; IORIO, R.C.; YAMAMURA, Y. Acupuntura no currículo médico: visão de estudantes de graduação em Medicina. Rev. Bras. Educ. Med., v.28, n.3, p.223-33, 2004.

AYRES, J. R. Epidemiologia, promoção da saúde e o paradoxo do risco. Rev. Bras. Epidemiol., v.5, supl.1, p.28-42, 2002.

BRASIL. Constituição da República Federativa do Brasil (1988). Brasília: Senado, 1988. Cap. 2, seção 2.

BRASIL. Ministério da Saúde. Política Nacional de Práticas Integrativas e

Complementares. Ministério da Saúde, Municipais de Saúde - Brasília: Ministério da Saúde, 2006. Disponível em: <http://dtr2004.saude.gov.br/dab/docs/publicacoes/geral/ pnpic.pdf>. Acesso em: 10 jun. 2009.

. Ministério da Saúde. Secretaria de Atenção à Saúde. Departamento de Atenção Básica. Política Nacional de Promoção de Saúde. Brasília: Ministério da Saúde, 2005. Disponível em: <http://bvsms.saude.gov.br/bvs/publicacoes/portaria687_2006_anexo1. pdf>. Acesso em: 10 jun. 2009.

Ministério da Saúde. Portaria no 1230/GM. Dispõe sobre a inclusão da consulta médica em homeopatia na tabela de procedimentos do SIA/SUS. Ministério da Saúde, Municipais de Saúde - Brasília: Ministério da Saúde, 1999.

. Ministério da Saúde. Portaria 971/06. Dispõe sobre a integralidade da atenção como diretriz do SUS e aprova a Política Nacional de Práticas Integrativas e Complementares (PNPIC) no Sistema Único de Saúde. Ministério da Saúde, Municipais de Saúde - Brasília: Ministério da Saúde, 2006. Disponível em: <http://www.ensp.fiocruz.br/ informe/anexos/Portaria\%20n971-2006.pdf>. Acesso em: 10 jun. 2009.

Ministério da Saúde. Prefeitura do Município de São Paulo. Secretaria Municipal de Saúde de São Paulo. Caderno Temático da Medicina Tradicional Chinesa, 2002. Disponível em: <http://ww2.prefeitura.sp.gov.br//arquivos/secretarias/saude/ publicacoes/0001/MTC_CadernoTem\%c3\%a1tico.pdf>. Acesso em: 10 jun. 2009. 
CAMARGO JR., K.R. A biomedicina. Physis, n.15, supl., p.177-201, 2005.

Biomedicina, saber e ciência: uma abordagem crítica. São Paulo: Hucitec, 2003.

CONSELHO NACIONAL DE SAÚDE - CNS (Brasil). Resolução 196/96, 1996. Diretrizes e Normas de Pesquisa em Seres Humanos. Disponível em: < http://www.ufrgs.br/bioetica/ res19696.htm >. Acesso em: 10 jun. 2009.

FIADEIROS, I. Lumaluma e ratatak. In: ALMEIDA, M.V. (Org.). Corpo presente - 13 reflexões antropológicas sobre o corpo. Oeiras: Celta, 1996. p.104-24.

FOUCAULT, M. O nascimento da clínica. Trad. Roberto Machado. 6.ed. Rio de Janeiro: Forense Universitária. 2008.

FUNDAMENTOS essenciais da Acupuntura chinesa. Trad. Sonia Regina de Lima Maike. São Paulo: Ícone, 1995.

GRANET, M. O pensamento chinês. Trad. Vera Ribeiro. Rio de Janeiro: Contraponto, 1997.

HICKS, A.; HICKS, J.; MOLE, P. Acupuntura constitucional dos cinco elementos. São Paulo: Roca, 2007

LALONDE, M. A new perspective on the health of Canadians. Ottawa; Minister of National Health and Welfare. 1974. Disponível em: <http://www.hc-sc.gc.ca/hcs-sss/ com/fed/lalonde-eng.php >. Acesso em: 7 ago. 2008.

LEAVELL, A.; CLARK, E.G. Medicina preventiva. São Paulo: McGraw Hill, 1976.

LEFÈVRE, F.; LEFÈVRE, A.M.C. Promoção de saúde: a negação da negação. Rio de Janeiro: Vieira \& Lent, 2004.

LUZ, D. Medicina tradicional chinesa, racionalidade médica. In: NASCIMENTO, M.C. (Org.). As duas faces da montanha: estudos sobre medicina chinesa e Acupuntura. São Paulo: Hucitec, 2006. p.83-141.

A medicina tradicional chinesa (MTC). Série Estudos em Saúde Coletiva, n.72, p.1-64, 1993

LUZ, M.T. Novos saberes e práticas em saúde coletiva. São Paulo: Hucitec, 2007. 1995.

Racionalidades médicas e terapêuticas alternativas. Cad. Sociol., v.7, p.108-28,

LUZ, M.T. et al. I seminário do projeto racionalidades médicas. Rio de Janeiro: IMSUERJ, 1992

LUZ, M.T.; SOUZA, E.F.A.A. Medical rationality contributions to research design in Chinese Medicine. Texto não publicado, 2009.

NASCIMENTO, M.C. Acupuntura, medicina e interculturalidade. In: (Org.). As duas faces da montanha: estudos sobre medicina chinesa e Acupuntura. São Paulo: Hucitec, 2006. p.143-77.

PEREIRA, I.M.T.B.; PENTEADO, R.Z.; MARCELO, V.C. Promoção de saúde e educação em saúde: uma parceria saudável. O mundo da saúde, v.24, n.1, p.39-44, 2000.

QUEIROZ, M.S. O sentido do conceito de medicina alternativa e movimento vitalista: uma perspectiva teórica introdutória. In: NASCIMENTO, M.C. (Org.). As duas faces da montanha: estudos sobre medicina chinesa e Acupuntura. São Paulo: Hucitec, 2006. p.19-39.

O itinerário rumo às medicinas alternativas: uma análise em representações sociais de profissionais de saúde. Cad. Saude Publica, v.16, p.363-74, 2000

SíCOLI, J.L.; NASCIMENTO, P.R. Promoção da saúde: concepções, princípios e operacionalização. Interface - Comunic., Saude, Educ., v.7, n.12, p.91-112, 2003. 
SOUZA, E.F.A.A. Nutrindo a vitalidade: questões contemporâneas sobre a Racionalidade Médica Chinesa e seu desenvolvimento histórico cultural. 2008. Tese (Doutorado) - Instituto de Medicina Social, Universidade do Estado do Rio de Janeiro, Rio de Janeiro. 2008.

UNSCHULD, P. Nan - Ching - the classic of difficult issues. Berkeley: University of California Press, 1986.

\section{5.}

Medicine in China: a history of ideas. Berkeley: University of California Press,

\section{WORLD HEALTH ORGANIZATION. WHO. Health promotion evaluation:}

recommendations to policymakers. European Working Group on Health Promotion Evaluation. Original: English. Copenhagen / Denmark: Regional Office For Europe. Scherfigsvej 8. EUR/ICP/IVST 0501 03. Unedited. EUR/HFA. Target 15. E60706, 1998. Disponível em: <http://www.infosihat.gov.my/artikelHP/bahanrujukan/HEandPenilaian/ HP\% 20Evaluation.pdf>. Acesso em: 07 ago. 2008.

WHO. Carta de Otawa, 1986. Disponível em: <www.saude.gov.br>. Acesso em: 10 jun. 2009. $\overline{\text { em: } 10}$ jun. 2009.

Declaração de Alma-Ata, 1978. Disponível em: <www.saude.gov.br>. Acesso

ZIGLIO, E. et al. Inversión en favor de la salud. In: CONFERÊNCIA MUNDIAL DE PROMOCIÓN DE LA SALUD, 5., 2000, México. Anais... México, 2000. p.1-51.

CINTRA, M.E.R.; FIGUEIREDO, R. Acupuntura y promoción de salud: posibilidades en el servicio público de salud. Interface - Comunic., Saude, Educ., v.14, n.32, p.139-54, jan./ mar. 2010.

El presente estudio analiza las posibles contribuciones de la Acupuntura aplicada en los servicios públicos de salud en el municipio de São Paulo, Brasil, para la Promoción de la Salud. Se utilizada el planteamiento cualitativo de investigación con el uso de entrevistas en profundidad. Los resultados señalan que la Acupuntura practicada en los servicios públicos permite un tránsito interdisciplinario integrando la percepción del individuo sobre sí mismo y su contexto, posibilitando cambios en las causas primarias de las enfermedades. Así el contacto con la Acupuntura practicada en los servicios de salud de la red municipal de São Paulo se muestra pasible de contribuir a el desarrollo de acciones de Promoción de Salud.

Palabras clave: Acupuntura. Promoción de salud. Sistema Único de Salud. 